\title{
Cerebral palsy: Basic Approach and Oral Medications
}

Abdulrahman M. Alshahrani

Consultant Neurologist, Riyadh, Saudi Arabia

Department of Internal Medicine (Neurology), College of Medicine, Shaqra University, Shaqra-11961, Saudi Arabia

https://doi.org/10.37881/1.211

https://orcid.org/0000-0001-8814-9333

\begin{abstract}
Cerebral Palsy is one of the devastating disorders in medicine. This relatively common problem occurs in babies, lasts for a lifetime and creates disability ranging from mild to severe. The lesion cannot be cured but the consequences can be minimized with proper treatment. The past decades have witnessed a great deal of change and improvements in the management of Cerebral Palsy Procedures have been modified to be more effective and at the same time minimize the impact of therapy, bracing and surgery on the child's life. However, this changing and increasing knowledge did not reach many parts of the world. This review aims to provide concise information on the basic aspects and approaches to treatment of musculoskeletal problems of children with Cerebral Palsy. it will be useful in the correction of problems that cause lifelong disability for millions of children worldwide.
\end{abstract}

\section{INTRODUCTION}

Cerebral palsy (CP) is a term used to describe a problem with movement and posture that makes certain activities difficult. Even though someone who has cerebral palsy has problems moving his or her muscles, this is not because there is something wrong with the muscles or nerves. These difficulties are caused because of problems in the brain. The patient might have had an injury to the brain, or had a brain that didn't develop properly. These problems can affect the way the brain controls movement and posture. Simply stated, "Cerebral" refers to the brain, and "Palsy" refers to muscle weakness/poor control. Although the brain itself will not get worse, people who have cerebral palsy will usually change over time. Sometimes they will get better, and some patients will stay the same. Occasionally they will get worse, usually because of contracture of the joints or changes in the muscle tone (1). Cerebral palsy is the most common chronic disability of childhood today. It is ubiquitous and it occurs all around the world. In developed nations, the incidence is about 1 to 2 per 1000 births. In spite of improved obstetrical and perinatal care, CP remains with us. As a result of injury to the brain, these children have motor defects which will affect them for their entire lifetime. Treatment often starts when they are infants, and continues throughout their life, even into adulthood. The child and his parents become the focus of treatment because you cannot treat the child without involving the parents as well. The team has to consist of the physician who will be the captain of the team. That physician can be a pediatrician, orthopaedic surgeon, physiatrist or even a neurologist, but they must take overall control of the child and make sure that all parameters of care are attended to. The team has to also include the occupational therapist, the speech therapist, the physical therapist, teachers and social workers (2).

\section{Definition}

Cerebral Palsy is a disorder of movement and posture that appears during infancy or early childhood. It is caused by nonprogressive damage to the brain before, during, or shortly after birth. CP is not a single disease but a name given to a wide variety of static neuromotor impairment syndromes occurring secondary to a lesion in the developing brain. The damage to the brain is permanent and cannot be cured but the consequences can be minimized. Progressive musculoskeletal pathology occurs in 
most affected children. The lesion in the brain may occur during the prenatal, perinatal, or postnatal periods. Any nonprogressive central nervous system (CNS) injury occurring during the first 2 years of life is considered to be CP $(2,3)$. CP was first described by the English physician Sir Francis William Little in 1861 and was known as Little's disease for a long time. Little thought that this condition was caused by neonatal asphyxia. Later, Sigmund Freud and other scientists challenged Little's idea and proposed that a variety of insults during pregnancy could damage the developing brain. Today, it is accepted that only approximately $10 \%$ of cases of CP can be attributed to neonatal asphyxia. The majority occurs during the prenatal period, and in most of the cases, a specific cause cannot be identified $(2,3)$.

\section{Who Does Cerebral Palsy Affect?}

It is estimated that two out of every 1,000 newborn children will develop cerebral palsy. And approximately $40 \%$ of those born with cerebral palsy will have a severe case. Right now, about 10,000 babies and infants are diagnosed with the condition each year. In 2002, the number of cerebral palsy cases in 8-year-old children was found to be one in 278. It is the most common motor disorder in children and is second only to autism as the most common disability in children (1).

\section{What Causes Cerebral Palsy?}

Cerebral Palsy does not have a single cause like chicken pox or measles. There are many reasons why someone might have cerebral palsy. An unborn child might have suffered a brain injury, an infection, or abnormal development of the brain tissue. These are called "prenatal" causes, meaning they happened before birth. These causes are responsible for about $70 \%$ of the cases of cerebral palsy. Another $20 \%$ of cerebral palsy cases are caused by a brain injury that takes place during the birthing process. In the United States, about $10 \%$ of children who have cerebral palsy got it after they were born. This is called "acquired cerebral palsy."(The figures are higher in underveloped countries.) Acquired cerebral palsy happens when there is brain damage during the first few months or years of life. This damage can be caused by brain infections, like bacterial meningitis or viral encephalitis. It can also be caused by a head injury usually from a motor vehicle accident, a fall, or child abuse during the first few years of life when the brain development is still taking place (1).

\section{Risk factors}

Risk factors associated with $\mathrm{CP}$ are grouped into prenatal, perinatal, and postnatal factors. Prematurity and low birth weights are the two most important risk factors in developed countries with high standards of obstetrical care. Postnatal risk factors additionally play a major role in other countries. A clear association exists between premature delivery and spastic diplegic. Low birth weight increases the risk. Rubella, herpes simplex, toxoplasma, and cytomegaloviruses cross the placenta to infect the fetus and have severe effects on the developing CNS. Eclampsia or other severe maternal illness hypothermia, hypoglycemia of the neonate cause a reduction in the levels of oxygen and nutrients available to the fetus or an increase in the levels of toxins or waste products, adversely affecting the developing CNS. Multiple pregnancies or breech presentation also can increase the risk. Excess of bilirubin resulting from the haemolytic disease of the newborn is clearly associated with CP. Babies who carry these risk factors should be under close supervision by a pediatric neurologist for signs suggestive of neuromotor developmental delay $(4,5)$.

\section{Pathological findings in the CNS}

Specific brain lesions related to CP can be identified in most of the cases. These lesions occur in regions that are particularly sensitive to disturbances in blood supply and are grouped under the term hypoxic ischemic encephalopathy. Five types of hypoxic ischemic encephalopathy exist; parasagittal cerebral injury, periventricular leukomalacia, focal and multifocal ischemic brain necrosis, status marmoratus and selective neuronal necrosis (6).

Clinical findings 
Children with Cerebarl Palsy present with three types of motor problems. The primary impairments of muscle tone, balance, strength and selectivity are directly related to the damage in the CNS. Secondary impairments of muscle contractures and deformities develop over time in response to the primary problems and musculoskeletal growth. Tertiary impairments are adaptive mechanisms and coping responses that the child develops to adapt to the primary and secondary problems. One typical example is gastrocnemius spasticity as a primary impairment leading to secondary ankle plantar flexion contracture and knee hyperextension in stance as an adaptive mechanism (6).

\section{Mechanism of the movement problems}

Abnormal muscle tone, disturbance of balance mechanisms, muscle weakness and loss of selective motor control lead to an inability to stretch muscles. Muscle weakness, spasticity, and contractures also result in abnormal skeletal forces which cause bone deformity as the child grows older. Muscles grow through stretch. Stretch occurs during active movement. When the child wants to play, he moves and stretches the muscles. This creates the necessary input for muscle growth. The child with $\mathrm{CP}$ cannot play because of pathological tone, weakness, poor selective control and abnormal balance. His muscles are not stretched and do not grow. The distal biarticular muscles are more affected because selective motor control is worse distally and the biarticular muscles are more abnormal than are the monoarticular muscles. The child with $\mathrm{CP}$ has abnormalities of muscle tone and reflexes, shows delay in developmental milestones, and presents with posture and movement problems. When he tries to move, muscle contractions cannot be effectively controlled. This is a result of many factors (7).

\section{Classification}

Cerebral Palsy encompasses a spectrum of motor disorders of varying tone, anatomical distribution and severity. Clinicians classify patients to describe the specific problem, to predict prognosis and to guide treatment. Classification is based on the change in muscle tone, anatomical region of involvement and severity of the problem. Classification provides a clearer understanding of the specific patient and directs management.

\section{Spastic Cerebral Palsy}

Spasticity is defined as an increase in the physiological resistance of muscle to passive motion. It is part of the upper motor neuron syndrome characterized by hyper-reflexia, clonus, extensor plantar responses and primitive reflexes. Spastic CP is the most common form of CP. Approximately $70 \%$ to $80 \%$ of children with CP are spastic. Spastic CP is anatomically distributed into three types (8).

\section{Hemiplegic}

With hemiplegic, one side of the body is involved with the upper extremity generally more affected than the lower. Seizure disorders, visual field deficits, astereognosis, and proprioceptive loss are likely. Twenty percent of children with spastic CP have hemiplegic. A focal traumatic, vascular, or infectious lesion is the cause in many cases. A unilateral brain infarct with posthemorrhagic porencephaly can be seen on magnetic resonance imaging (MRI).

\section{Diplegic}

With diplegic, the lower extremities are severely involved and the arms are mildly involved. Intelligence usually is normal, and epilepsy is less common. Fifty per cent of children with spastic CP have diplegic. A history of prematurity is usual. Diplegic is becoming more common as more lowbirth-weight babies survive. MRI reveals mild periventricular leukomalacia (PVL).

Quadriplegic (Total body involvement - tetraplegic) With quadriplegic, all four limbs, the trunk and muscles that control the mouth, tongue, and pharynx are involved. When one upper extremity is less involved, the term triplegic is used. Thirty percent of children with spastic CP have quadriplegic. More serious involvement of lower extremities is common in premature babies. Some have perinatal hypoxic ischemic encephalopathy. MRI reveals PVL. 


\section{Dyskinetic Cerebral Palsy}

Abnormal movements that occur when the patient initiates movement are termed dyskinesias. Dysarthria, dysphagia, and drooling accompany the movement problem. Mental status is generally normal, however severe dysarthria makes communication difficult and leads the observer to think that the child has intellectual impairment. Sensorineural hearing dysfunction also impairs communication. Dyskinetic CP accounts for approximately $10 \%$ to $15 \%$ of all cases of CP. Hyperbilirubinemia or severe anoxia causes basal ganglia dysfunction and results in dyskinetic $\mathrm{CP}$.

\section{Ataxic Cerebral Palsy}

Ataxia is loss of balance, coordination, and fine motor control. Ataxic children cannot coordinate their movements. They are hypotonic during the first 2 years of life. Muscle tone becomes normal and ataxia becomes apparent toward the age of 2 to 3 years. Children who can walk have a wide-based gait and a mild intention tremor (dysmetria). Dexterity and fine motor control is poor. Ataxia is associated with cerebellar lesions.

\section{Mixed Cerebral Palsy}

Children with a mixed type of CP commonly have mild spasticity, dystonia, and/or athetoid movements. Ataxia may be a component of the motor dysfunction in patients in this group. Ataxia and spasticity often occur together. Spastic ataxic diplegic is a common mixed type that often is associated with hydrocephalus.

\section{Exceptions}

Some children with $\mathrm{CP}$ cannot be fitted into these $\mathrm{CP}$ groups because they present with many different impairments. Dystonia may be seen in the spastic child, and anatomical classification may not be fully explanatory because clinical findings may overlap. An example is the hypotonic total-body-involved baby who stays hypotonic throughout childhood. Define the pathological abnormalities observed in these children according to the anatomical, and clinical involvement, as described above (8).

\section{Oral Medications (9-11)}

Various pharmacological agents decrease spasticity. Baclofen, benzodiazepines (diazepam, clonazepam), dantrolene sodium and tizanidine are commonly used in children.

\section{Indications}

Consider systemic oral antispastic drugs in total body involved nonambulatory children with generalized spasticity. They are also useful for short periods after orthopaedic surgery. Systemic side effects such as drowsiness, sedation, and generalized weakness are common, so they generally are not recommended for ambulatory children. Keep the initial dose low and gradually titrate to a level at which the effect is maximal and the side effects are minimal. The responses of children to oral antispastic drugs are not consistent. Try different drugs to achieve a satisfactory clinical effect.

\section{Oral antispastic drugs Baclofen}

Baclofen is an agonist of the main inhibitory CNS neurotransmitter gamma aminobutyric acid (GABA). It shows its effect mainly on the spinal cord. It decreases spasticity by increasing the inhibitory effect of the interneuron on the alpha motor neuron. The lipid solubility of baclofen is poor, so it cannot easily cross the blood brain barrier. High oral doses are necessary to achieve a therapeutic dose in the cerebrospinal fluid (CSF). The effect starts 1 hour after ingestion and lasts for 8 hours. The drug must be taken three to four times daily in divided doses. Daily dose for children between ages 2 to 7 is 10 to 15 mgrs per day with a maximum of 40 mgrs per day. After the age of 8 years, the dose may be increased to 60 mgrs per day. Maximum doses range between 80 to $120 \mathrm{mg}$. per day in adults. Side effects including sleepiness, sedation, drowsiness, fatigue, headache, nausea, and a decrease in seizure threshold are commonly associated with increasing doses. Baclofen also causes generalised muscle weakness. All side effects are dose dependent. Sudden withdrawal may cause hallucinations and seizures sometimes 
accompanied by extreme hyperthermia and increased spasticity called the baclofen withdrawal syndrome. The dose of the drug must be decreased gradually.

\section{Diazepam}

Diazepam is a benzodiazepine tranquilizer that works as a GABA agonist. It enhances the presynaptic inhibitory effect of GABA and decreases spasticity. It is absorbed faster than baclofen, acts faster, and has a longer lasting effect. Doses in children range between 0.12 to $0.8 \mathrm{mg} / \mathrm{kg}$ body weight with a maximum of 20 mg. daily divided into two or three equal doses. Diazepam decreases painful muscular spasms and improves sleep. Sedation and other CNS side effects are very common, so this drug is not recommended for treating ambulatory children except after orthopaedic surgery when it improves the child's tolerance and participation in the rehabilitation program. CNS side effects are weakness, memory loss, ataxia, depression, and dependency.

\section{Clonazepam}

Clonazepam has an effect similar to that of diazepam, but it has a slightly longer half-life. It is preferred over diazepam because its side effects are fewer. Initial dose is 0.1 to $0.2 \mathrm{mg} / \mathrm{kg} /$ day. This dose is titrated for an optimal effect.

\section{Dantrolene sodium}

Dantrolene sodium inhibits muscle contraction by blocking calcium release from the sarcoplasmic reticulum in the muscle fiber. Initial dose is 0.5 $\mathrm{mg} / \mathrm{kg}$ of body weight with a maximum dose of 3 $\mathrm{mg} / \mathrm{kg}$ of body weight. Total daily dose should not exceed $12 \mathrm{mg}$ per day administered in four divided doses. Side effects include muscle weakness, sedation, diarrhoea, and hepatotoxicity. CNS side effects are rare. Liver function tests should be performed two to four times a year, and the total treatment duration should not exceed 2 years.

\section{Tizanidine}

Tizanidine is an alpha adrenergic receptor agonist. It shows its effect at the brain and the spinal cord level. Tizanidine decreases the release of excitatory neurotransmitters and increases the release of inhibitory neurotransmitters. Guidelines for use in children are not well established. In adults the initial dose is 2 to $4 \mathrm{mg}$. administered at 4 hour intervals and increased to $36 \mathrm{mg}$. as needed. It may cause drowsiness, nausea, hallucinations, and is hepatotoxic (9-11).

\section{Neuromuscular Blocking Agents}

Local Anesthetics, Phenol, Botulinum Toxin Consider using local anesthetics, alcohol, phenol and botulinum toxin as neuromuscular blocking agents when treating focal spasticity.

\section{Local anesthetics}

\section{Mechanism of effect}

Local anesthetics block nerve conduction by changing membrane permeability to sodium ions. They affect both sensory and motor function in the area innervated by the nerve. This effect is completely reversible and causes no structural damage to the nerve. The effect starts within 3-15 minutes after the injection and lasts from 45 minutes to 8-12 hours depending on the type of drug used. Median nerve in the upper extremity and many nerves in the lower extremity are available for local anesthetic blocks.

\section{Dosing and administration}

Lidocaine, etidocaine and bupivacaine are used for nerve blocks. Prefer bupivacaine because it is more potent and its duration of action is longer. It can be injected in amounts up to $3 \mathrm{mg} / \mathrm{kg}$ of 0.25 to $0.75 \%$ of a solution. Do a perineural injection when you want to block the motor, sensory and autonomic fibers in the nerve. A motor point block affects the motor fibers only. A peripheral nerve stimulator that gives a low intensity electrical current through a needle electrode is used for blocks. Use small needles and give short-lasting stimuli to localize the nerve more accurately. This makes the procedure less painful.

\section{Indications}

Local anesthetic blocks may be used as a diagnostic tool to differentiate spasticity from contracture and to predict functional changes with long term therapy. 
The block may clarify which muscles contribute to spasticity and unmask selective motor control in the antagonist muscles if there is any. Block the median nerve at the elbow to evaluate the upper extremity. If flexion in the wrist and fingers is due to spasticity, the hand will relax completely a couple of minutes after the injection. Bring the fingers into extension while holding the wrist in extension. The joint will not relax if there is a contracture. Thus, a local anesthetic block aids the physician in the decision making process of treatment of the spastic hand.

\section{Advantages}

Local anesthetics have a short and reversible effect, so they are useful for diagnosis of the problem and differentiating contracture from dynamic spasticity.

\section{Side effects and precautions}

Local anesthetics rarely cause a hypersensitivity reaction in the form of a mild rash. Fatal anaphylactoid reactions have been reported. Hematoma may occur at the injection site. There can be significant changes in walking and transfers after a nerve block. Sudden decrease in muscle tone may result in falls and injuries in the early hours after the block. In high doses, local anesthetics may have systemic toxic side effects if they enter the systemic circulation by mistake. This is uncommon in children and in doses used for peripheral nerve blocks.

\section{Botulinum toxin}

Botulinum toxin, produced by the anaerobic bacteria Clostridium botulinum, is one of the most potent poisons known to man. In the past two decades it has been transformed into one of the most useful antispastic agents. Of the seven distinct toxins from $A$ to $G$, only type $A$ and $B$ are used for therapeutic purposes. The structure of all toxins and their mechanism of action are similar, only their site of action is different $(12,13)$.

\section{The mechanism of effect}

The toxin inhibits acetylcholine release at the neuromuscular junction causing a reversible chemodenervation. Studiessuggest that the toxin affects the muscle spindle and afferent nerve fibers as secondary actions. Effect at the neuromuscular junction The toxin must enter the nerve endings to exert its effect. It becomes fully active once inside the cholinergic nerve terminal. When the impulse for contraction arrives at the axon terminal acetylcholine (Ach) vesicles fuse with the nerve membrane and the Ach is released into the synaptic cleft. This causes excitation in the muscle fiber and muscle contraction. The various serotypes of botulinum toxin act on different portions of the acetylcholine vesicle complex. Botulinum toxin inhibits the fusion of acetylcholine vesicles at the pre-synaptic membrane. Ach cannot be released into the synaptic cleft, the impulse from the nerve to the muscle fiber is blocked and the muscle fibers innervated by that axon cannot contract. This is chemical denervation. The extent of muscle weakness created by the botulinum toxin depends on the serotype, dose and volume of toxin used. The effect of botulinum toxin is reversible. Nerve sprouts form at the unmyelinated terminal axon immediately proximal to the end plate. These sprouts innervate the muscle fiber. Eventually, the original neuromuscular junction regains function. This terminates the clinical effect in 3 - 6 months and spasticity reappears (14).

Afferent effect: The toxin may block the sensory afferents from the muscle spindle. This reduces spindle sensitivity and consequent reflex action.

Analgesic effect: There is an analgesic effect of the toxin explained by a couple of mechanisms. First, decreasing spasticity decreases pain. Second, botulinum toxin affects afferent transmission and inhibits the release of substance P. Substance $P$ is the primary mediator of pain in the spinal cord and the brain. Inhibition of its release together with the block in afferent transmission result in pain relief (14).

\section{Indications}

Botulinum toxin injections have been used as a safe and effective treatment for spastic $\mathrm{CP}$ for the past 10 years. Botulinum toxin $\mathrm{B}$ is also becoming commercially available. The general indication for botulinum toxin injections in $\mathrm{CP}$ is 'the presence of a 
dynamic contracture, interfering with function, in the absence of a fixed muscular contracture'. If botulinum toxin injections are started at an early age and repeated as necessary, they can help prevent the development of muscle contractures and bony deformities. This helps to delay orthopaedic surgery until the gait is mature. The need for extensive surgical procedures may be eliminated if bony deformities are prevented by botulinum toxin. The success of botulinum toxin administration depends on many factors. Patient selection is critical. Children with spasticity who do not have fixed contractures benefit a great deal from treatment whereas patients with dyskinesia have a variable response and athetoids do not benefit at all. The timing of the injections is controversial. Most clinicians agree that the earlier the spasticity is reduced, the better the outcome. Botulinum toxin can be injected as early as 18 months of age. There is no upper age limit, however, once the muscle is shortened as occurs with age, the effect of spasticity relief will not be apparent because of contracture (15).

\section{Dosing and administration}

Botulinum toxin dosing depends on which preparation is used. Dysport dosing is different than Botox and there is no equivalence ratio between the two preparations in terms of clinical effect. The doses mentioned here refer to Botox injections. The amount changes according to the number of muscles to be treated, prior response of the patient if there are any prior injections and functional goals. The dose limits range from 2 units to 29 units/kg of body weight, most common range being between 10-20 units $/ \mathrm{kg}$ of body weight. Avoid injecting more than 400 to 600 units of total dose at any one time, injecting more than 50 units at one injection site and exceeding 20 units per kilogram per muscle at any one time. If there is a need for more toxin because of multilevel involvement, combine treatment with phenol. Inject larger muscles with phenol and use botulinum toxin for more distal and smaller muscles. Targeting the neuromuscular junction during the injection using electrical stimulation guide may result in more effect for less volume. Even though no serious complications have been reported, it is a good idea to apply high doses under general anesthesia in the operating theatre. Reduce the dose if the child is small and has atrophic muscles, if the treatment is going to be repeated for a number of times and if multiple muscles are being injected. Severely spastic and larger muscles should receive a larger dose whereas less spastic and small muscles receive a smaller dose (16-18). The amount of toxin given to one muscle must be divided into more than two injection sites, depending on the dose. Put a safe distance between two injection sites with high doses. This increases the diffusion of the toxin in the muscle and prevents it from entering the systemic circulation. Divide the total dose per muscle over more sites as much as possible. For example, for a 20 kg child who has a very spastic gastrocnemius muscle, the dose should be $6 \mathrm{U} / \mathrm{kg} /$ muscle, $120 \mathrm{U}$ totals. This dose should be divided into 4 injection sites, 30 units per site in the muscle (19-24).

\section{Conclusion}

More information about the causes of cerebral palsy is likely to come from further exploration of the role of infection in the perinatal period, investigations of the role of coagulation and inflammatory factors and the use of sophisticated brain imaging. By classifying the various types of cerebral palsy according to their clinical features, it may be possible to determine factors unique to particular motor disorders and to infants born at different gestations. Ongoing research about both risk factors and causes and newer medication and approaches in treatment in cerebral palsy will be an essential first step in the important goal of developing strategies for prevention.

\section{References:}

1. https://ethnomed.org/patienteducation/neurological-conditions/cerebralpalsy 
2. Rosenbaum P, Paneth N, Leviton A et al. A report: the definition and classification of cerebral palsy. April 2006, Dev Med Child Neurol Suppl, 2007; 109: 8-14.

3. Russman BS. Cerebral Palsy: Definition, Manifestations and Etiology. Turk J Phys Med Rehabil. 2002; 48 (2): 4-6.

4. Cans C, McManus V, Crowley $\mathrm{M}$ et al. Surveillance of Cerebral Palsy in Europe Collaborative Group, Cerebral palsy of postneonatal origin: characteristics and risk factors. Paediatr Perinat Epidemiol. 2004; 18(3):214-20.

5. Han TR, Bang MS, Lim JY et al. Risk factors of cerebral palsy in preterm infants. Am J Phys Med Rehabil. 2002, 81(4):297-303.

6. Blasco PA. Pathology of cerebral palsy In The Diplegic Child: Evaluation and Management. Sussman MD AAOS, Rosemont. 1992; 3-20.

7. Dormans JP,Copley LA. Musculoskeletal impairments In Caring for Children with Cerebral Palsy A Team Approach. Dormans JP, Pellegrino L, Paul H Brookes Co Baltimore. 1998; 125-141.

8. Panteliadis CP. Classification in Cerebral Palsy: Principles and Management. Panteliadis CP, Strassburg HM Stuttgart Thieme. 2004.

9. Scheinberg A, Hall K, Lam LT,et al. Oral baclofen in children with cerebral palsy: a double-blind cross-over pilot study. J Paediatr Child Health. 2006; 42(11):715-20.

10. Verrotti A, Greco R, Spalice A et al. Pharmacotherapy of spasticity in children with cerebral palsy. Pediatr Neurol. 2006; 34(1):1-6.

11. Elovic E. Principles of pharmaceutical management of spastic hypertonia. Phys Med Rehabil Clin N Am. 2001; 12(4):793-816.

12. Heinen F, Desloovere K, Schroeder AS et al. The updated European Consensus 2009 on the use of Botulinum toxin for children with cerebral palsy. Eur J Paediatr Neurol. 2010; 14(1):45-66.

13. Lannin N, Scheinberg A, Clark K. AACPDM systematic review of the effectiveness of therapy for children with cerebral palsy after botulinum toxin A injections. Developmental Medicine \& Child Neurology. 2006; 48: 533-9.

14. Ackman JD, Russman BS, Thomas SS et al. Comparing botulinum toxin A with casting for treatment of dynamic equinus in children with cerebral palsy. Developmental Medicine and Child Neurology. 2005; 47:620-627.

15. Berweck S, Heinen F. Use of botulinum toxin in pediatric spasticity (cerebral palsy). Mov Disord. 2004; 19 Suppl 8:S162-7.

16. Gooch JL, Patton CP. Combining botulinum toxin and phenol to manage spasticity in children Arch Phys Med Rehabil. 2004; 85(7):1121-4.

17. Boyd RN, Hays RM. Outcome measurement of effectiveness of botulinum toxin type $\mathrm{A}$ in children with cerebral palsy: an ICIDH-2 approach. Eur J Neurol 8 Suppl. 2001; 5:16777.

18. Koman LA, Brashear A, Rosenfeld S et al. Botulinum toxin type a neuromuscular blockade in the treatment of equinus foot deformity in cerebral palsy: a multicenter open-label clinical trial. Pediatrics. 2001; 108(5):1062-71.

19. Zafonte RD, Munin MC. Phenol and alcohol for the treatment of spasticity. Phys Med Rehabil Clin N Am. 2001; 12(4):817-832.

20. Molenaers G, Desloovere K, De Cat J et al. Single event multilevel botulinum toxin type A treatment and surgery: Similarities and differences. Eur J Neurol. 2001; 8(5):88-97.

21. Molenaers G, Desloovere K, Eyssen M, et al. Botulinum toxin type A treatment of cerebral palsy: An integrated approach. Eur J Neurol. 1999; 6(4):S51-S57. 


\section{NeuroPharmac Journal}

Journal homepage: www.neuropharmac.com
ISSN:2456-3927

Review Article
22. Wissel J, Heinen F, Schenkel A, et al. Botulinum toxin $A$ in the management of spastic gait disorders in children and young adults with cerebral palsy: A randomized, double-blind study of 'high-dose' versus 'lowdose' treatment. Neuropediatrics. 1999; 30(3):120-124

23. MF Brin. Botulinum Toxin: Chemistry, Pharmacology, Toxicity, and Immunology Muscle Nerve. 1997; 20 (6): S146-S168.

24. Chutorian A, Root L, BTA Study Group A multi-centered, randomized, double- blind placebo-controlled trial of botulinum toxin type $\mathrm{A}$ in the treatment of lower limb spasticity in pediatric cerebral palsy. Mov Disord. 1995; 10:364.

\section{Copyright}

(C) 2017 NeuroPharmac J. This is an open access journal, and articles are distributed under the terms of the Creative Commons AttributionNonCommercial-ShareAlike 4.0 License.

Cite this article: Alshahrani A. Cerebral palsy: Basic Approach and Oral Medications. NeuroPharmac J. 2017; 2(1): 32-40. DOI: $10.37881 / 1.211$ 